\title{
Examples of genus two fibrations with no sections on rational surfaces
}

\author{
By Shinya KitagawA \\ General Education (Natural Sciences), National Institute of Technology, Gifu College, \\ 2236-2 Kamimakuwa, Motosu, Gifu 501-0495, Japan \\ (Communicated by Shigefumi MORI, M.J.A., Oct. 12, 2017)
}

\begin{abstract}
We construct explicit examples of genus two fibrations with no sections on rational surfaces by the double covering method. For the proof of non-existence of sections, we use the theory of the virtual Mordell-Weil groups.
\end{abstract}

Key words: Sections of fibrations; Mordell-Weil groups; rational surfaces.

1. Introduction. We shall work over the complex number field C. Let $S$ be a smooth projective rational surface and $\varphi: S \rightarrow \mathbf{P}^{1}$ a fibration whose general fibre $F$ is a projective curve of genus $g \geq 1$. We assume that $\varphi$ is relatively minimal, i.e., there are no $(-1)$-curves contained in fibres. In the case where $g=1$, it is well-known that the Picard number $\rho(S)$ equals ten. Furthermore, as a consequence of the canonical bundle formula of Kodaira [10, Theorem 12], $\varphi$ admits a section if and only if $\varphi$ does not have any multiple fibres (e.g., [4, Proposition (1.1.)]). When $g \geq 2$, it can be shown that $11 \leq \rho(S) \leq 4 g+6$ by a similar argument to $[7, \S 2]$ (cf. [9], see also [14, Theorem 2.8] and [3]). Furthermore, if either $\rho(S)=4 g+6$ with $g \neq 1$ or $\rho(S)=13$ with $g=2$ holds, then $\varphi$ always admits a section (cf. [8, Theorem 2.2] and [6, Lemma 1.4], see also [14], [13] and $[2, \S 10.5])$.

We restrict ourselves to the case where $g=2$ and $\rho(S)=12$. Suppose that $\varphi$ admits a section. We can regard it as a horizontal curve $D$ on $S$ such that $D . F=1$. Therefore the fibres of $\varphi$ contain at least one irreducible component with multiplicity one (cf. Remark 5). The purpose of the paper is to construct examples for the converse:

Theorem 1. Let $(t, x)$ be local coordinates of $\left(\mathbf{P}^{1} \backslash\{\infty\}\right) \times\left(\mathbf{P}^{1} \backslash\{\infty\}\right)$ and $p r_{1}: \mathbf{P}^{1} \times \mathbf{P}^{1} \rightarrow \mathbf{P}^{1}$ the projection map onto the first factor. Put $\Gamma_{0}=$ $p r_{1}^{-1}(0)$ and $\Gamma_{\infty}=p r_{1}^{-1}(\infty)$. Let $\sigma$ and $\tau$ be complex numbers with $\sigma \neq 0,1$ and $\tau \neq 0,1 / 2,2 / 3,1$. Let $A(\gamma, \tau)$ denote the closure of the zero set of a polynomial $\gamma \tau x^{3}-\gamma t x^{2}-(3 \tau-2) t x+(2 \tau-1) t^{2}$ in $t, x$ on $\mathbf{P}^{1} \times \mathbf{P}^{1}$ for $\gamma \in \mathbf{C} \backslash\{0\}$. Put $B=A(1, \tau)+$

2010 Mathematics Subject Classification. Primary 14D06; Secondary 14G05, 14J26.
$A(\sigma, \tau)+\Gamma_{0}+\Gamma_{\infty}$. Let $\pi: \hat{X} \rightarrow \mathbf{P}^{1} \times \mathbf{P}^{1}$ be the finite double cover branched along $B$ and $\mu: \tilde{X} \rightarrow \hat{X}$ the canonical resolution of singularities of $\hat{X}$. Then $a$ general fibre of $p r_{1} \circ \pi \circ \mu: \tilde{X} \rightarrow \mathbf{P}^{1}$ is a smooth curve of genus two. Let $f: X \rightarrow \mathbf{P}^{1}$ be the relatively minimal model of $p r_{1} \circ \pi \circ \mu$.

Then $X$ is a smooth rational surface with $\rho(X)=12$. Furthermore, $f: X \rightarrow \mathbf{P}^{1}$ has no sections, the fibres $f^{-1}(0)$ and $f^{-1}(\infty)$ are as in $\left[11\right.$, p. 172, 3-II-0 $\mathrm{I}_{3-0}$, and the other fibres of $f$ are irreducible and reduced. In particular, the fibres of $f$ contain at least one irreducible component with multiplicity one.

Let us explain the organization of the paper. At first we construct a genus two fibration $f: X \rightarrow \mathbf{P}^{1}$ as in Theorem 1 from a $\mathbf{P}^{1}$-bundle $\Sigma_{0}$ with a given branch divisor by the double covering method. In Proposition 3, we show the assertions as in Theorem 1 except for non-existence of a section of $f$. If $f$ has a section, then $f$ has at least two sections since the branch divisor does not contain a section of $\Sigma_{0}$ (cf. Lemma 6$)$.

Next we consider the ruling associated to $2 K_{X}+F$, where $K_{X}$ denotes the canonical divisor. The degenerate fibres consist of the $(-2)$-curves contained in fibres of $f$ and the $(-1)$-bisections of $f$. Here a $(-1)$-bisection of $f$ means a $(-1)$-curve meeting $F$ at two points. In Proposition 7 , through a birational morphism from $X$ to a relatively minimal model of the ruling, we have an explicit description of the Néron-Severi group $\operatorname{NS}(X)$. Then we see that there is a (non-effective) divisor $D$ with $D . F=1$. In fact, Corollary 8 gives us that $D$ and the irreducible components of the fibres of $f$ generate $\mathrm{NS}(X)$.

In $\S 3$, we introduce the theory of the virtual 
Mordell-Weil groups. Further, Corollary 8 yields that the virtual Mordell-Weil group of $f$ is trivial. As a result, a section of $f$ is unique if it exists. This contradicts Lemma 6 . Therefore $f$ has no sections. This completes the proof of Theorem 1 .

2. Construction. In this section we shall construct a smooth projective rational surface together with a relatively minimal fibration of genus two as in Theorem 1. Furthermore, we shall describe singular fibres of the fibration and the Néron-Severi group of the surface, which coincides with the Picard group in our situation.

Put $\Sigma_{0}=\mathbf{P}^{1} \times \mathbf{P}^{1}$. Denote by $(t, x)$ the inhomogeneous coordinates on $\Sigma_{0}$. Let $p r_{n}: \Sigma_{0} \rightarrow \mathbf{P}^{1}$ be the projection map onto the $n$-th factor. Put $\Gamma_{q}=$ $p r_{1}^{-1}(q)$ and $\Delta_{q}=p r_{2}^{-1}(q)$ for any point $q \in \mathbf{P}^{1}$. Let $\sigma$ and $\tau$ be complex numbers with $\sigma \neq 0,1$ and $\tau \neq 0,1 / 2,1$. Let $A(\gamma, \tau)$ denote the closure of the zero set of a polynomial $\gamma \tau x^{3}-\gamma t x^{2}-(3 \tau-2) t x+$ $(2 \tau-1) t^{2}$ in $t, x$ on $\mathbf{P}^{1} \times \mathbf{P}^{1}$ for $\gamma \in \mathbf{C} \backslash\{0\}$. Put $B=A(1, \tau)+A(\sigma, \tau)+\Gamma_{0}+\Gamma_{\infty}$.

Let $\phi_{1}: W_{1} \rightarrow \Sigma_{0}$ be the blow-up at two points $(0,0)$ and $(\infty, \infty)$ with the exceptional curves $E_{0,1}$ and $E_{\infty, 1}$, i.e., $\phi_{1}\left(E_{0,1}\right)=(0,0)$ and $\phi_{1}\left(E_{\infty, 1}\right)=$ $(\infty, \infty)$. Let $P_{i, 2}$ be the intersection point of $E_{i, 1}$ and the strict transform to $W_{1}$ of $\Gamma_{i}$ for $i=0, \infty$. The strict transform $A_{1}(\gamma, \tau)$ to $W_{1}$ of $A(\gamma, \tau)$ passes through the two points $P_{0,2}$ and $P_{\infty, 2}$. The local intersection number at $P_{0,2}$ of $A_{1}(1, \tau)$ and $A_{1}(\sigma, \tau)$ is two if and only if $\tau=2 / 3$. Next let $\phi_{2}: W_{2} \rightarrow W_{1}$ be the blow-up at two points $P_{0,2}$ and $P_{\infty, 2}$ with $E_{0,2}=\phi_{2}^{-1}\left(P_{0,2}\right)$ and $E_{\infty, 2}=\phi_{2}^{-1}\left(P_{\infty, 2}\right)$. The strict transform to $W_{2}$ of $\Delta_{0}$ and that of $\Delta_{\infty}$ are $(-1)$-curves on $W_{2}$. Let $\phi_{2}^{\prime}: W_{2} \rightarrow W_{1}^{\prime}$ be the contraction of the two $(-1)$-curves. The image by $\phi_{2}^{\prime}$ of the strict transform to $W_{2}$ of $\Gamma_{0}$ and that of $\Gamma_{\infty}$ are $(-1)$-curves on $W_{1}^{\prime}$. By contracting them, we get another $\mathbf{P}^{1} \times \mathbf{P}^{1}$ and denote it by $\Sigma_{0}^{\prime}$. Then the images of $E_{0,2}$ and $E_{\infty, 2}$ by the other contraction $\phi^{\prime}: W_{2} \rightarrow \Sigma_{0}^{\prime}$ are two distinct fibres of the projection map $\mathrm{pr}_{2}^{\prime}: \Sigma_{0}^{\prime} \rightarrow \mathbf{P}^{1}$ onto the second factor. Similarly, the images by $\phi^{\prime}$ of the strict transforms of $E_{0,1}$ and $E_{\infty, 1}$ are two distinct fibres of the projection map $\mathrm{pr}_{1}^{\prime}: \Sigma_{0}^{\prime} \rightarrow \mathbf{P}^{1}$ onto the first factor. Therefore we may assume that the strict transform to $W_{2}$ of $\Gamma_{i}$ contracts by $\phi^{\prime}$ to the point $(i, i)$ for $i=0, \infty$.

Lemma 2. Let $\phi^{\prime}: W_{2} \rightarrow \Sigma_{0}^{\prime}$ be the above contraction. Denote by $(v, z)$ the inhomogeneous coordinates on $\Sigma_{0}^{\prime}$. Let $\psi: \Sigma_{0} \rightarrow \Sigma_{0}^{\prime}$ be the rational map given by $(v, z)=\left(t / x^{2}, t / x\right)$. Then $\psi$ is the birational map satisfying $\phi^{\prime}=\psi \circ \phi_{1} \circ \phi_{2}$. Furthermore, the closure of the zero set of a polynomial $\gamma(\tau-z)-(3 \tau-2) v+(2 \tau-1) v z$ in $v, z$ on $\Sigma_{0}^{\prime}$ is isomorphic to the strict transform $A_{2}(\gamma, \tau)$ to $W_{2}$ of $A(\gamma, \tau)$ for $\gamma \in \mathbf{C} \backslash\{0\}$. In particular, $A(\gamma, \tau)$ is irreducible and tangent to $\Gamma_{1 / \gamma}$ at the point given by $(t, x)=(1 / \gamma, 1 / \gamma)$, and $B$ is reduced.

Proof. We obtain $\psi^{-1}$ by setting $(t, x)=$ $\left(z^{2} / v, z / v\right)$. The indeterminacy of $\psi^{-1}$ consists of $(0,0),(\infty, \infty)$ and their infinitely near points. Let $A^{\prime}(\gamma, \tau)$ denote the closure of the zero set of a polynomial $\gamma(\tau-z)-(3 \tau-2) v+(2 \tau-1) v z$ in $v, z$ on $\Sigma_{0}^{\prime}$. From $\tau \neq 1$, we show that $A^{\prime}(\gamma, \tau)$ is irreducible. In fact, $A^{\prime}(\gamma, \tau)$ is tangent to the closure of the zero set of a polynomial $v-\gamma z^{2}$ in $v, z$ on $\Sigma_{0}^{\prime}$ at $(\gamma, 1)$. Therefore $A(\gamma, \tau)$ is tangent to $\Gamma_{1 / \gamma}$ at $(1 / \gamma, 1 / \gamma)$. It follows from $\tau \neq 0,1 / 2$ that $A^{\prime}(\gamma, \tau)$ does not pass through $(0,0),(\infty, \infty)$. Thus $A^{\prime}(\gamma, \tau)$ is isomorphic to $A_{2}(\gamma, \tau)$ through $\phi^{\prime}$. In particular, $A(\gamma, \tau)$ is also irreducible and $B$ is reduced.

Notice that $B$ is divisible by two in the Picard group $\operatorname{Pic}\left(\Sigma_{0}\right)$ of $\Sigma_{0}$. Since $\operatorname{Pic}\left(\Sigma_{0}\right)$ is torsion free, there is a unique element $\delta \in \operatorname{Pic}\left(\Sigma_{0}\right)$ with $B \sim 2 \delta$, where the symbol $\sim$ means the linear equivalence of divisors. Thus a finite double cover of $\Sigma_{0}$ branched along $B$ is uniquely constructed from $B$ up to isomorphism. We denote it by $\pi: \hat{X} \rightarrow \Sigma_{0}$. Let us resolve singularities of $\hat{X}$ according to Horikawa [5]. In fact, $\Gamma_{0}$ and $\Gamma_{\infty}$ are singular fibres of type $(\mathrm{V})$ as in [5, p. 84, Definition] for all $\sigma, \tau$ with $\sigma \neq 0,1$ and $\tau \neq 0,1 / 2,1$. Furthermore, $W_{2}$ coincides with $W^{b}$ as in [5, p. 85, Lemma 7]. In our situation singularities of the induced branch divisor $B^{b}$ are two simple triple points, which correspond to the point $(\infty,(3 \tau-2) /(2 \tau-1))$ and the point $(0, \tau)$ on $\Sigma_{0}^{\prime}$ through $\phi^{\prime}$ as in Lemma 2. On $W_{2}$, in the corresponding points, $A_{2}(1, \tau)$ and $A_{2}(\sigma, \tau)$ meet transversally. One of the two points, which is denoted by $P_{0,3}$, is on the strict transform of $E_{0,1}$. We denote by $P_{\infty, 3}$ the other one, which is on the strict transform of $E_{\infty, 1}$. Denote by $\phi_{3}: W_{3} \rightarrow W_{2}$ the blow-up at the two points $P_{0,3}$ and $P_{\infty, 3}$ with $E_{0,3}=\phi_{3}^{-1}\left(P_{0,3}\right)$ and $E_{\infty, 3}=\phi_{3}^{-1}\left(P_{\infty, 3}\right)$. Let $A_{3}(\gamma, \tau)$ denote the strict transform to $W_{3}$ of $A(\gamma, \tau)$. Remark that $A_{3}(1, \tau)$ and $A_{3}(\sigma, \tau)$ are disjoint from each other.

Let $P_{i, 4}$ denote the intersection point of $E_{i, 3}$ and $A_{3}(1, \tau)$ and let $P_{i, 5}$ be that of $E_{i, 3}$ and $A_{3}(\sigma, \tau)$ for $i=0, \infty$. Denote by $P_{i, 6}$ the intersection point of $E_{i, 3}$ and the strict transform to $W_{3}$ of $E_{i, 1}$. Let $\phi_{6}$ : 
$\tilde{W} \rightarrow W_{3}$ be the blow-up at the six points $P_{i, j}$ with $i=0, \infty$ and $j=4,5,6$. Set $E_{i, j}=\phi_{6}^{-1}\left(P_{i, j}\right)$. For $i=$ $0, \infty$ and $k=1,2,3$, we denote by $\hat{E}_{i, k}$ the strict transform to $\tilde{W}$ of $E_{i, k}$. In the same way, $\hat{\Delta}_{i}$ and $\hat{\Gamma}_{i}$ denote respectively that of $\Delta_{i}$ and $\Gamma_{i}$. Let $A_{6}(\gamma, \tau)$ be the strict transform to $\tilde{W}$ of $A(\gamma, \tau)$. For simplicity, we denote the pull-back to $\tilde{W}$ of them by the same symbols. Thus we have $A_{6}(1, \tau)+$ $E_{0,4}+E_{\infty, 4} \sim A_{6}(\sigma, \tau)+E_{0,5}+E_{\infty, 5} \sim 3 \Delta_{0}+2 \Gamma_{0}-$ $2 E_{0,1}-E_{0,2}-E_{0,3}-2 E_{\infty, 1}-E_{\infty, 2}-E_{\infty, 3}$.

Now, we set

$$
\begin{aligned}
\tilde{B}= & A_{6}(1, \tau)+A_{6}(\sigma, \tau)+\hat{\Gamma}_{0}+\hat{\Gamma}_{\infty} \\
& +\hat{E}_{0,1}+\hat{E}_{0,3}+\hat{E}_{\infty, 1}+\hat{E}_{\infty, 3} .
\end{aligned}
$$

Since $\tilde{B}$ is smooth and divisible by two in $\operatorname{Pic}(\tilde{W})$, we obtain a smooth projective surface $\tilde{X}$ by the finite double cover $\varpi: \tilde{X} \rightarrow \tilde{W}$ branched along $\tilde{B}$. Put $\tilde{\phi}=\phi_{1} \circ \phi_{2} \circ \phi_{3} \circ \phi_{6}$. Then there exists the birational morphism $\mu: \tilde{X} \rightarrow \hat{X}$ with $\pi \circ \mu=$ $\tilde{\phi} \circ \varpi$. We call $\tilde{X}$ the canonical resolution of singularities of $\hat{X}$. For simplicity, we put $\tilde{\pi}=\pi \circ \mu$ and $\tilde{f}=p r_{1} \circ \tilde{\pi}$.

Let us consider $\tilde{f}: \tilde{X} \rightarrow \mathbf{P}^{1}$. We set $q(\gamma, \tau)=$ $\tau(3 \tau-2)^{3} /(2 \gamma \tau-\gamma)$ for two complex numbers $\gamma$ and $\tau$ with $\gamma \neq 0$ and $\tau \neq 0,1 / 2,1$. If $\tau \neq 2 / 3$, then $\Gamma_{q(\gamma, \tau)}$ is tangent to $A(\gamma, \tau)$. We know from Lemma 2 that $\Gamma_{1 / \gamma}$ does so. By restricting $p r_{1} \circ \tilde{\phi}: \tilde{W} \rightarrow \mathbf{P}^{1}$ to $A_{6}(1, \tau)$ and to $A_{6}(\sigma, \tau)$, we see that $\Gamma_{q}$ meets $B$ transversely at six points for any $q \in \mathbf{P}^{1} \backslash\{0, \infty, 1$, $1 / \sigma, q(1, \tau), q(\sigma, \tau)\}$ from the Riemann-Hurwitz formula. Therefore a general fibre of $\tilde{f}: \tilde{X} \rightarrow \mathbf{P}^{1}$ is a smooth projective curve of genus two. Furthermore, we remark that $q(1, \tau)-1=(3 \tau-1)^{3}(\tau-1) /(2 \tau-$ 1) and $\Gamma_{1}$ meets $A(\sigma, 1 / 3)$ transversely at three points. Exactly at two points $\Gamma_{1}$ meets $B$ transversely if and only if $\sigma$ and $\tau$ satisfy $q(\sigma, \tau)=1$. When $q(1, \tau) \neq 1$ and $q(\sigma, \tau) \neq 1$, at four points $\Gamma_{1}$ meets $B$ transversely. Thus $\Gamma_{1}$ meets $B$ transversely at least at two points. In this way, we can check that $\Gamma_{1 / \sigma}, \Gamma_{q(1, \tau)}$ and $\Gamma_{q(\sigma, \tau)}$ also do so. Hence the reducible fibres of $\tilde{f}$ are $\tilde{f}^{-1}(0)$ and $\tilde{f}^{-1}(\infty)$ only.

Let $e_{i}$ denote the $(-1)$-curve on $\tilde{X}$ with $2 e_{i}=$ $\varpi^{*} \hat{\Gamma}_{i}$ for $i=0, \infty$. Although $e_{i}$ meets $\varpi^{*} \hat{E}_{i, 2}$ at one point, $e_{i}$ is disjoint from the other components of $\tilde{f}^{-1}(i)$. Additionally, $\varpi^{*} \hat{E}_{i, 2}$ is not a $(-2)$-curve. Thus, after the contraction $\eta: \tilde{X} \rightarrow X$ of $e_{0}$ and $e_{\infty}$, we obtain the relatively minimal model $f: X \rightarrow \mathbf{P}^{1}$ of $\tilde{f}: \tilde{X} \rightarrow \mathbf{P}^{1}$.

Proposition 3. For two complex numbers $\sigma$ and $\tau$ with $\sigma \neq 0,1$ and $\tau \neq 0,1 / 2,1$, the fibration $f: X \rightarrow \mathbf{P}^{1}$ obtained as above is a relatively minimal fibration of genus two. The fibre $f^{-1}(\infty)$ is as in $\left[11\right.$, p. $\left.172,3-\mathrm{II}_{3-0}^{*}\right]$. If $\tau \neq 2 / 3$, then $f^{-1}(0)$ is also as in $\left[11\right.$, p. $\left.172,3-\mathrm{II}_{3-0}^{*}\right]$. However, $f^{-1}(0)$ is as in $[11$, p. $155,1-\mathrm{IV}]$ if $\tau=2 / 3$. The other fibres of $f$ are irreducible and reduced. Furthermore, $X$ is a smooth rational surface with $\rho(X)=12$.

Proof. Consider $A_{1}(1, \tau)+A_{1}(\sigma, \tau)+E_{0,1}+$ $E_{\infty, 1}$ on $W_{1}$. For $i=0, \infty$, the strict transform to $W_{1}$ of $\Gamma_{i}$ is a $(-1)$-curve. The contraction of the two (-1)-curves translates singularities of the branch divisor into those as in [11, p. 155, 1-IV] and $\left[11\right.$, p. $\left.172,3-\mathrm{II}_{3-0}^{*}\right]$. Thus, it is enough to prove that $\tilde{X}$ is a rational surface with $\rho(\tilde{X})=14$.

Consider the projection map $p r_{1}^{\prime}: \Sigma_{0}^{\prime} \rightarrow \mathbf{P}^{1}$ onto the first factor. Let $\Gamma_{1}^{\prime}$ be the pull-back to $\tilde{W}$ of the fibre given by $v=1$ on $\Sigma_{0}^{\prime}$. We remark that $\varpi^{*} \Gamma_{1}^{\prime}$ is nef. Let us compute $\varpi^{*} \Gamma_{1}^{\prime} \cdot K_{\tilde{X}}$. Lemma 2 yields $\varpi^{*} \Gamma_{1}^{\prime} \sim \varpi^{*}\left(2 \Delta_{0}+\Gamma_{0}-E_{0,1}-E_{0,2}-E_{\infty, 1}-\right.$ $\left.E_{\infty, 2}\right)$. We know

$$
\begin{aligned}
\tilde{B} \sim & 6 \Delta_{0}+6 \Gamma_{0}-4\left(E_{0,1}+E_{0,2}+E_{\infty, 1}+E_{\infty, 2}\right) \\
& -2 \sum_{j=3}^{6}\left(E_{0, j}+E_{\infty, j}\right) .
\end{aligned}
$$

Hence $K_{\tilde{X}} \sim \varpi^{*}\left(K_{\tilde{W}}+\tilde{B} / 2\right) \sim \varpi^{*}\left(\Delta_{0}+\Gamma_{0}-E_{0,1}-\right.$ $\left.E_{0,2}-E_{\infty, 1}-E_{\infty, 2}\right)$. For all positive integers $n$, we conclude that the $n$-th plurigenus of $\tilde{X}$ is zero from $\varpi^{*} \Gamma_{1}^{\prime} \cdot n K_{\tilde{X}}=-2 n<0$. The finite double cover $\varpi$ also gives us $\chi(\tilde{X})=2 \chi(\tilde{W})+\tilde{B} \cdot K_{\tilde{W}} / 4+\tilde{B}^{2} / 8=1$ (e.g., $[1$, p. 237]), where $\chi(\tilde{X})$ and $\chi(\tilde{W})$ respectively denote the Euler characteristic of $\tilde{X}$ and $\tilde{W}$. This implies that the irregularity of $\tilde{X}$ is zero. Therefore $\tilde{X}$ is a rational surface by Castelnuovo's rationality criterion. Thus $b_{1}(\tilde{X})=b_{3}(\tilde{X})=0$ and $b_{2}(\tilde{X})=$ $\rho(\tilde{X})$, where $b_{n}(\tilde{X})$ denotes the $n$-th Betti number of $\tilde{X}$. This and Noether's formula provide $\rho(\tilde{X})=$ $10-K_{\tilde{X}}^{2}$. So $\rho(\tilde{X})=14$ follows.

Corollary 4. Keep the notation and assumptions as above. For $i=0, \infty$ and $j=1,3$, let $\Theta_{i, j}$ denote the (-2)-curve with $2 \eta^{*} \Theta_{i, j}=\varpi^{*} \hat{E}_{i, j}$. Let $\Theta_{i, j}$ be the (-2)-curve which is identified with $\varpi^{*} E_{i, j}$ through $\eta$ for $i=0, \infty$ and $j=4,5,6$. Put $\Theta_{i, 2}=$ $\eta\left(\varpi^{*} \hat{E}_{i, 2}\right)$ for $i=0, \infty$. Then $\Theta_{0,2}$ is a $(-3)$-curve with $\Theta_{0,2} \cdot \Theta_{0,3}=1$ if $\tau=2 / 3$ and an elliptic curve with $\Theta_{0,2} \cdot \Theta_{0,1}=-\Theta_{0,2}^{2}=1$ otherwise. $\Theta_{\infty, 2}$ is an elliptic curve with $\Theta_{\infty, 2} \cdot \Theta_{\infty, 1}=-\Theta_{\infty, 2}^{2}=1$ for every

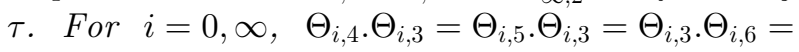
$\Theta_{i, 6} \cdot \Theta_{i, 1}=1$. For the other pairs of irreducible components of the fibres, two components are 
disjoint from each other. In particular, the dual graph of the configuration of $\Theta_{i, 4}, \Theta_{i, 5}, \Theta_{i, 3}, \Theta_{i, 6}$ and $\Theta_{i, 1}$ has the Dynkin diagram of type $D_{5}$. Furthermore, the irreducible decompositions of the two reducible fibres of $f$ are as follows: $f^{-1}(0)=2 \Theta_{0,2}+$ $6 \Theta_{0,3}+3 \Theta_{0,4}+3 \Theta_{0,5}+4 \Theta_{0,6}+2 \Theta_{0,1}$ if $\tau=2 / 3$ and $f^{-1}(0)=\Theta_{0,4}+\Theta_{0,5}+2 \Theta_{0,3}+2 \Theta_{0,6}+2 \Theta_{0,1}+2 \Theta_{0,2}$ otherwise. $\quad f^{-1}(\infty)=\Theta_{\infty, 4}+\Theta_{\infty, 5}+2 \Theta_{\infty, 3}+$ $2 \Theta_{\infty, 6}+2 \Theta_{\infty, 1}+2 \Theta_{\infty, 2}$ for every $\tau$.

Remark 5. Let $f: X \rightarrow \mathbf{P}^{1}$ be a fibration with $\tau=2 / 3$ as in Proposition 3. Then a nonexistence result for sections can be derived from an easy calculation as follows: Suppose that $f$ admits a section. We can regard it as a horizontal curve $D_{0}$ on $X$ such that $D_{0} \cdot f^{-1}(q)=1$ for all $q \in \mathbf{P}^{1}$. However, $D_{0} \cdot f^{-1}(0) \geq 2$ since multiplicities of the irreducible components of $f^{-1}(0)$ are at least two. We obtain a contradiction.

In order to prove that $f$ has no sections for every $\tau$, we need the following

Lemma 6. Let $f: X \rightarrow \mathbf{P}^{1}$ be a fibration as in Proposition 3. If there exists a section of $f$, then $f$ has at least two sections.

Proof. Suppose that there exists a section of $f$, which can be regarded as a horizontal curve $D_{1}$ on $X$. Let $\tilde{D}_{1}$ be the strict transform by $\eta$ of $D_{1}$. Then the image $C$ by $\tilde{\pi}$ of $\tilde{D}_{1}$ is a section of $p r_{1}: \Sigma_{0} \rightarrow \mathbf{P}^{1}$. Here we recall the irreducible decomposition of $B$. In particular, $B$ does not contain $C$ as an irreducible component. Hence we obtain another section $\tilde{D}_{2}$ of $\tilde{f}$ as the other component $\left(\tilde{\pi}^{*} C-\tilde{D}_{1}\right)$ from the irreducible decomposition of $\tilde{\pi}^{*} C$. So we have another section of $f$ as the image by $\eta$ of $\tilde{D}_{2}$.

To describe irreducible components of fibres of $f: X \rightarrow \mathbf{P}^{1}$ in $\mathrm{NS}(X)$, through a birational morphism $X \rightarrow \mathbf{P}^{2}$, we interpret $f: X \rightarrow \mathbf{P}^{1}$ as a pencil generated by plane curves of degree seven which have one triple point and ten double points at eleven base points.

Proposition 7. Let $f: X \rightarrow \mathbf{P}^{1}$ be a fibration as in Proposition 3, $F$ a general fibre of $f$ and $\Theta_{i, j}$ the irreducible components of the reducible fibres as in Corollary 4. Then there exists a birational morphism $\nu: X \rightarrow \mathbf{P}^{2}$ such that the pull-back to $X$ of a line $\ell$ on $\mathbf{P}^{2}$ and that of $(-1)$-curves $e_{h}, h=1,2, \ldots, 11$ contracted by $\nu$ satisfy the following

(a) $F=7 \ell-3 e_{1}-2 \sum_{h=2}^{11} e_{h}, \quad \Theta_{0,4}=\ell-e_{1}-$ $e_{8}-e_{10}, \Theta_{\infty, 4}=\ell-e_{1}-e_{9}-e_{11}$,

$$
\begin{array}{ll}
\Theta_{0,1}=e_{4}-e_{2}, & \Theta_{\infty, 1}=e_{5}-e_{3}, \\
\Theta_{0,6}=e_{6}-e_{4}, & \Theta_{\infty, 6}=e_{7}-e_{5}, \\
\Theta_{0,3}=e_{8}-e_{6}, & \Theta_{\infty, 3}=e_{9}-e_{7}, \\
\Theta_{0,5}=e_{10}-e_{8}, & \Theta_{\infty, 5}=e_{11}-e_{9}
\end{array}
$$

and $\Theta_{\infty, 2}=-K_{X}+e_{3}$ for every $\tau$.

(b) $\Theta_{0,2}=2 \ell-e_{3}-e_{5}-e_{7}-e_{8}-e_{9}-e_{10}-e_{11}$ if $\tau=2 / 3$ and $\Theta_{0,2}=-K_{X}+e_{2}$ otherwise.

Proof. Let $e_{1}$ denote the $(-1)$-curve with $2 \eta^{*} e_{1}=\varpi^{*} A_{6}(1, \tau)$. Since $\Gamma_{1} \cdot A_{6}(1, \tau)=3$ on $\tilde{W}$, we have $F \cdot e_{1}=3$. The images by $\eta$ of $\varpi^{*} \hat{\Delta}_{0}$ and $\varpi^{*} \hat{\Delta}_{\infty}$ are two disjoint $(-1)$-curves on $X$, which meet $F$ at two points. Put $e_{2}=\eta\left(\varpi^{*} \hat{\Delta}_{0}\right)$ and $e_{3}=\eta\left(\varpi^{*} \hat{\Delta}_{\infty}\right)$. We also remark that $e_{2} \cdot e_{1}=e_{3} \cdot e_{1}=0$, since $\hat{\Delta}_{0} \cdot A_{6}(1, \tau)=\hat{\Delta}_{\infty} \cdot A_{6}(1, \tau)=0$.

We know $\varpi^{*} \Gamma_{1}^{\prime} \cdot K_{\tilde{X}}=-2$ in the proof of Proposition 3. By the adjunction formula we verify that $p r_{1}^{\prime}: \Sigma_{0}^{\prime} \rightarrow \mathbf{P}^{1}$ induces a ruling $\tilde{\xi}: \tilde{X} \rightarrow \mathbf{P}^{1}$ through $\psi \circ \tilde{\pi}: \tilde{X} \rightarrow \Sigma_{0}^{\prime}$. The degenerate fibres of $\tilde{\xi}$ are $\tilde{\xi}^{-1}(0)$ and $\tilde{\xi}^{-1}(\infty)$ only, since the fibres of $p r_{1}^{\prime}$ except for $p r_{1}^{\prime-1}(0)$ and $p r_{1}^{\prime-1}(\infty)$ do not pass through the intersection points of $A^{\prime}(1, \tau)$ and $A^{\prime}(\sigma, \tau)$. Recall the definition of $\psi$ in Lemma 2. Then $\tilde{\xi}^{-1}(i)$ contains $e_{i}$ for each $i=0, \infty$ as an irreducible component. Hence there is a unique ruling $\xi: X \rightarrow$ $\mathbf{P}^{1}$ such that $\tilde{\xi}=\xi \circ \eta$. Furthermore, $\xi^{-1}(\infty)$ consists of $\Theta_{0, j}, j=1,3,4,5,6$ and $e_{2}$ whose configuration is as in [8, Figure 2]. In a similar configuration as above, $\xi^{-1}(0)$ consists of $\Theta_{\infty, j}, j=1,3,4,5,6$ and $e_{3}$.

Notice that $e_{1} \cdot \Theta_{0,4}=e_{1} \cdot \Theta_{\infty, 4}=1$ and the other components are disjoint from $e_{1}$. Let $\nu_{1}: X \rightarrow \Sigma_{1}$ be the birational morphism contracting $e_{2}, e_{3}$ and eight $(-2)$-curves $\Theta_{i, 1}, \Theta_{i, 6}, \Theta_{i, 3}, \Theta_{i, 5}$ with $i=0, \infty$, which are the strict transforms of eight $(-1)$-curves. Here $\Sigma_{1}$ is a relatively minimal model of $\xi: X \rightarrow \mathbf{P}^{1}$. We remark that the image of $e_{1}$ is the minimal section of $\Sigma_{1}$. Let $\nu_{0}: \Sigma_{1} \rightarrow \mathbf{P}^{2}$ be the contraction and put $\nu=\nu_{0} \circ \nu_{1}$.

From the configuration of $e_{2}, e_{3}$ and eight $(-2)$-curves $\Theta_{i, 1}, \Theta_{i, 6}, \Theta_{i, 3}, \Theta_{i, 5}$ with $i=0, \infty$, we can denote the pull-backs to $X$ of the eight (-1)-curves by $e_{h}, h=4,5, \ldots, 11$ so that $e_{4}=$ $\Theta_{0,1}+e_{2}, e_{5}=\Theta_{\infty, 1}+e_{3}, e_{6}=\Theta_{0,6}+e_{4}, e_{7}=\Theta_{\infty, 6}+$ $e_{5}, e_{8}=\Theta_{0,3}+e_{6}, e_{9}=\Theta_{\infty, 3}+e_{7}, e_{10}=\Theta_{0,5}+e_{8}$ and $e_{11}=\Theta_{\infty, 5}+e_{9}$. We recall that the images by $\nu_{1}$ of $\Theta_{0,4}$ and $\Theta_{\infty, 4}$ are fibres of $\Sigma_{1}$. It follows from the configurations of $\xi^{-1}(0)$ and $\xi^{-1}(\infty)$ that $\Theta_{0,4}=$ $\ell-e_{1}-e_{8}-e_{10}$ and $\Theta_{\infty, 4}=\ell-e_{1}-e_{9}-e_{11}$, where $\ell$ denotes the pull-back to $X$ of a line on $\mathbf{P}^{2}$. 
Next we recall that $\varpi^{*} \hat{\Delta}_{0}=\eta^{*} e_{2}-e_{\infty}$ and $K_{\tilde{X}} \sim \varpi^{*}\left(K_{\tilde{W}}+\tilde{B} / 2\right) \sim \varpi^{*} \hat{\Delta}_{0}-\varpi^{*} E_{0,2}+\varpi^{*} \hat{\Gamma}_{\infty}$. Hence we have $\varpi^{*} E_{0,2} \sim-K_{\tilde{X}}+\eta^{*} e_{2}+e_{\infty} \sim$ $\eta^{*}\left(-K_{X}+e_{2}\right)-e_{0}$. Therefore we get $\Theta_{0.2}=-K_{X}+$ $e_{2}$ from $\hat{E}_{0,2}=E_{0,2}$ when $\tau \neq 2 / 3$. In the same way, we see $\Theta_{\infty, 2}=-K_{X}+e_{3}$ for every $\tau$. Furthermore, we show $F \sim f^{-1}(\infty)=2 \Theta_{\infty, 2}+2 \Theta_{\infty, 1}+2 \Theta_{\infty, 6}+$ $2 \Theta_{\infty, 3}+\Theta_{\infty, 4}+\Theta_{\infty, 5} \sim 7 \ell-3 e_{1}-2 \sum_{h=2}^{11} e_{h}$.

To complete the proof, it is enough to describe $\Theta_{0,2}$ by the $\mathbf{Z}$-linear combinations of $\ell$ and $e_{h}$ 's when $\tau=2 / 3$. The description follows immediately from the others as in the condition (a) and the irreducible decomposition of $f^{-1}(0)$ as in Corollary 4.

Corollary 8. Put $D=e_{1}-e_{11}$. Then

$$
\mathrm{NS}(X) \simeq \mathbf{Z} D \oplus \bigoplus_{j=1}^{6} \mathbf{Z} \Theta_{0, j} \oplus \bigoplus_{j=1}^{4} \mathbf{Z} \Theta_{\infty, j} \oplus \mathbf{Z} \Theta_{\infty, 6} .
$$

Proof. It is well-known that $\mathrm{NS}(X) \simeq$ $\mathbf{Z} \ell \oplus \bigoplus_{h=1}^{11} \mathbf{Z} e_{h}$. Thus we only have to represent $\ell$ and $e_{h}$ 's as Z-linear combinations of $D$ and $\Theta_{i, j}$ 's from Proposition 7. Suppose $\tau \neq 2 / 3$. Then $D$ and the irreducible components of the reducible fibres except for $\Theta_{\infty, 5}$ generate $\ell$ and $e_{1}$ as follows:

$$
\begin{aligned}
\ell= & 7 D+16 \Theta_{0,1}+13 \Theta_{0,2}+22 \Theta_{0,3}+14 \Theta_{0,4} \\
& +11 \Theta_{0,5}+19 \Theta_{0,6}-13 \Theta_{\infty, 1}-16 \Theta_{\infty, 2} \\
& -7 \Theta_{\infty, 3}-4 \Theta_{\infty, 4}-10 \Theta_{\infty, 6}, \\
e_{1}= & 3 D+6 \Theta_{0,1}+5 \Theta_{0,2}+8 \Theta_{0,3}+5 \Theta_{0,4}+4 \Theta_{0,5} \\
& +7 \Theta_{0,6}-5 \Theta_{\infty, 1}-6 \Theta_{\infty, 2}-3 \Theta_{\infty, 3}-2 \Theta_{\infty, 4} \\
& -4 \Theta_{\infty, 6} .
\end{aligned}
$$

Furthermore, Proposition 7 immediately yields that

$$
\begin{aligned}
& e_{11}=e_{1}-D, e_{9}=\ell-e_{1}-e_{11}-\Theta_{\infty, 4}, \\
& e_{7}=e_{9}-\Theta_{\infty, 3}, \quad e_{5}=e_{7}-\Theta_{\infty, 6}, e_{3}=e_{5}-\Theta_{\infty, 1}, \\
& e_{2}=e_{3}+\Theta_{0,2}-\Theta_{\infty, 2}, e_{4}=e_{2}+\Theta_{0,1}, \\
& e_{6}=e_{4}+\Theta_{0,6}, e_{8}=e_{6}+\Theta_{0,3}, e_{10}=e_{8}+\Theta_{0,5} .
\end{aligned}
$$

Hence, we see that $e_{h}, h=2,3, \ldots, 11$ can be the Z-linear combinations inductively.

In the case of $\tau=2 / 3$, we only have to replace $\varpi^{*} E_{0,2}=\Theta_{0,2}$, which holds if $\tau \neq 2 / 3$, with $\varpi^{*} E_{0,2}=\Theta_{0,2}+2 \Theta_{0,3}+\Theta_{0,4}+\Theta_{0,5}+\Theta_{0,6}$. Therefore $D, \quad \Theta_{0,1}, \Theta_{0,2}, \ldots, \Theta_{0,6}, \quad \Theta_{\infty, 1}, \Theta_{\infty, 2}, \Theta_{\infty, 3}, \Theta_{\infty, 4}$ and $\Theta_{\infty, 6}$ form Z-basis of $\operatorname{NS}(X)$ for every $\tau$.

3. Virtual Mordell-Weil groups. We shall introduce the theory of the virtual MordellWeil groups, which is used to complete the proof of Theorem 1. Let $S$ be a smooth projective rational surface and $\varphi: S \rightarrow \mathbf{P}^{1}$ a relatively minimal fibra- tion whose general fibre $F$ is a projective curve of genus $g \geq 2$. We denote by $n_{q}$ the number of irreducible components of $\varphi^{-1}(q)$ for any $q \in \mathbf{P}^{1}$. In [12, Definition 0.2], the virtual Mordell-Weil rank $r$ of $\varphi$, which does not necessarily admit a section, is defined as $r=\rho(S)-2-\sum_{q \in \mathbf{P}^{1}}\left(n_{q}-1\right)$. For example, when $\varphi: S \rightarrow \mathbf{P}^{1}$ is a fibration as in Proposition 3 , we know that $\rho(S)=12$ and $n_{0}=n_{\infty}=6$ with $n_{q}=1$ for all $q \in \mathbf{P}^{1} \backslash\{0, \infty\}$, which lead to $r=0$.

Let $V$ be the subgroup of $\mathrm{NS}(S)$ generated by the irreducible components of the fibres of $\varphi$. The primitive closure $\hat{V}$ is defined as $\hat{V}=V \otimes \mathbf{Q} \cap$ $\mathrm{NS}(S)$. Let us observe that $\hat{V} / V$ can be regarded as the virtual Mordell-Weil group of $\varphi$ with $r=0$. Assume that there exists on $S$ a divisor $D$ with $D . F=1$. When $\varphi$ admits a section, we can regard it as the above $D$. Set $T_{D}=\mathbf{Z} D \oplus V \subset \operatorname{NS}(S)$. The primitive closure $\hat{T}_{D}$ is defined as $\hat{T}_{D}=T_{D} \otimes \mathbf{Q} \cap$ $\operatorname{NS}(S)$. Then, for all $D$ with $D \cdot F=1$, the quotient groups of $\hat{T}_{D}$ by $T_{D}$ are isomorphic to each other and to $\hat{V} / V$ as follows:

Lemma 9. The natural projection $T_{D} \rightarrow V$ induces an isomorphism $\hat{T}_{D} / T_{D} \simeq \hat{V} / V$ naturally.

Proof. Let $\Theta_{q, j}$ be an irreducible component of a reducible fibre $\varphi^{-1}(q)$ for $j=1,2, \ldots, n_{q}$. Take any $E \in \hat{T}_{D}$. We have $E=\alpha D+\sum \beta_{q, j} \Theta_{q, j}$ for some $\alpha, \beta_{q, j} \in \mathbf{Q}$ by definition. In fact, $\alpha=E . F$ must be an integer. In particular, $\sum \beta_{q, j} \Theta_{q, j}=E-\alpha D \in$ $\mathrm{NS}(S)$. Therefore, $\sum \beta_{q, j} \Theta_{q, j} \in \hat{V}$. In this way, while $D . F=1$, the natural projection $\hat{T}_{D} \rightarrow \hat{V}$ of the primitive closures is well-defined. Consider the composition $\hat{T}_{D} \rightarrow \hat{V} \rightarrow \hat{V} / V$, which is a surjective homomorphism of groups. Its kernel is equal to $T_{D}$. So $\hat{T}_{D} / T_{D} \simeq \hat{V} / V$ follows.

Via $\varphi$, we can regard $S$ as a smooth projective curve of genus $g$ defined over the rational function field $\mathbf{K}=\mathbf{C}\left(\mathbf{P}^{1}\right)$. We assume that it has a $\mathbf{K}$-rational point $O$. Let $\mathcal{J}_{\varphi} / \mathbf{K}$ be the Jacobian variety of the generic fibre $\mathcal{F} / \mathbf{K}$ of $\varphi$. The Mordell-Weil group of $\varphi$ is the group of $\mathbf{K}$-rational points $\mathcal{J}_{\varphi}(\mathbf{K})$. It is a finitely generated abelian group. The rank of the group is equal to $r$. There is a natural one-to-one correspondence between the set of $\mathbf{K}$-rational points $\mathcal{F}(\mathbf{K})$ and the set of sections of $\varphi$. For $P \in \mathcal{F}(\mathbf{K})$, we denote by $(P)$ the section corresponding to $P$ which is regarded as a horizontal curve on $S$. In particular, $(O)$ corresponding to the origin $O$ of $\mathcal{J}_{\varphi}(\mathbf{K})$ is called the zero section. In $[15$, Theorem 3$]$, we have the natural isomorphism 
of groups $\mathcal{J}_{\varphi}(\mathbf{K}) \simeq \mathrm{NS}(S) / T_{(O)}$. By definition, the torsion part $\mathcal{J}_{\varphi}(\mathbf{K})_{\text {tor }}$ is isomorphic to $\hat{T}_{(O)} / T_{(O)}$. From Lemma 9, we conclude $\mathcal{J}_{\varphi}(\mathbf{K})_{\text {tor }} \simeq \hat{V} / V$.

Let $f: X \rightarrow \mathbf{P}^{1}$ be a fibration as in Proposition 3. We recall $r=0$. Furthermore, if $f$ admits a section, then we see that the Mordell-Weil group of $f$ is trivial from Corollary 8 and Lemma 9. Hence, we have the following

Lemma 10. Let $f: X \rightarrow \mathbf{P}^{1}$ be a fibration as in Proposition 3. Suppose that $f$ admits a section. Then it is the unique section of $f$.

Proof of Theorem 1. Let $f: X \rightarrow \mathbf{P}^{1}$ be a fibration as in Proposition 3. We only have to show that $f$ has no sections. The assertion follows from Lemmas 6 and 10.

Acknowledgements. The author would like to express his heartfelt gratitude to Prof. Kazuhiro Konno for his constant encouragement and kind advice. The author would also like to thank Profs. Yoichi Imayoshi and Takeshi Harui for inspiring interests in fibrations without sections. Thanks are also due to Profs. Tadashi Ashikaga, Atsushi Ikeda and Hirotaka Ishida for interesting discussions.

\section{References}

[ 1 ] W. P. Barth, K. Hulek, C. A. M. Peters and A. Van de Ven, Compact complex surfaces, 2nd ed., Ergebnisse der Mathematik und ihrer Grenzgebiete. 3. Folge. A Series of Modern Surveys in Mathematics, 4, Springer-Verlag, Berlin, 2004.

[ 2 ] A. Calabri and C. Ciliberto, Birational classification of curves on rational surfaces, Nagoya Math. J. 199 (2010), 43-93.

[ 3 ] M. de Franchis, Riduzione dei fasci di curve piane di genere 2, Rend. Circ. Mat. Palermo 13 (1899), 1-27.

[ 4 ] Y. Fujimoto, On rational elliptic surfaces with multiple fibers, Publ. Res. Inst. Math. Sci. 26 (1990), no. 1, 1-13.

[ 5 ] E. Horikawa, On algebraic surfaces with pencils of curves of genus 2, in Complex analysis and algebraic geometry, Iwanami Shoten, Tokyo, 1977, pp. 79-90.

[ 6 ] S. Kitagawa and K. Konno, Fibred rational surfaces with extremal Mordell-Weil lattices, Math. Z. 251 (2005), no. 1, 179-204.

[ 7 ] S. Kitagawa, Maximal Mordell-Weil lattices of fibred surfaces with $p_{g}=q=0$, Rend. Semin. Mat. Univ. Padova 117 (2007), 205-230.

[ 8 ] S. Kitagawa, Extremal hyperelliptic fibrations on rational surfaces, Saitama Math. J. 30 (2013), $1-14$.

[ 9 ] S. Kitagawa, Pencils of genus two curves on rational surfaces, arXiv:1006.4372.

[ 10 ] K. Kodaira, On the structure of compact complex analytic surfaces. I, Amer. J. Math. 86 (1964), 751-798.

[11] Y. Namikawa and K. Ueno, The complete classification of fibres in pencils of curves of genus two, Manuscripta Math. 9 (1973), 143-186.

[ 12 ] K. V. Nguyen, On upperbounds of virtual Mordell-Weil ranks, Osaka J. Math. 34 (1997), no. 1, 101-114.

[ 13 ] K. V. Nguyen, On certain Mordell-Weil lattices of hyperelliptic type on rational surfaces, J. Math. Sci. (New York) 102 (2000), no. 2, 3938-3977.

[14 ] M.-H. Saito and K.-I. Sakakibara, On MordellWeil lattices of higher genus fibrations on rational surfaces, J. Math. Kyoto Univ. 34 (1994), no. 4, 859-871.

[ 15 ] T. Shioda, Mordell-Weil lattices for higher genus fibration over a curve, in New trends in algebraic geometry (Warwick, 1996), 359-373, London Math. Soc. Lecture Note Ser., 264, Cambridge Univ. Press, Cambridge, 1999. 